\title{
Gas and dust properties in the afterglow spectra of GRB 050730^
}

\author{
R. L. C. Starling ${ }^{1}$, P. M. Vreeswijk ${ }^{2}$, S. L. Ellison ${ }^{3}$, E. Rol ${ }^{4}$, K. Wiersema ${ }^{1}$, A. J. Levan ${ }^{4,5}$, N. R. Tanvir ${ }^{5}$, \\ R. A. M. J. Wijers ${ }^{1}$, C. Tadhunter ${ }^{6}$, J. R. Zaurin ${ }^{6}$, R. M. Gonzalez Delgado ${ }^{7}$, and C. Kouveliotou ${ }^{8}$
}

\author{
1 Astronomical Institute "Anton Pannekoek", University of Amsterdam, Kruislaan 403, 1098 SJ Amsterdam, The Netherlands \\ e-mail: starling@science. uva.nl \\ 2 European Southern Observatory, Alonso de Córdova 3107, Casilla 19001, Santiago 19, Chile \\ 3 Dept. of Physics and Astronomy, University of Victoria, Elliott Building, 3800 Finnerty Rd, Victoria, BC, V8P 1A1, Canada \\ 4 Dept. of Physics and Astronomy, University of Leicester, University Road, Leicester LE1 7RH, UK \\ 5 Centre for Astrophysics Research, University of Hertfordshire, College Lane, Hatfield, Herts. AL10 9AB, UK \\ ${ }^{6}$ Dept. of Physics and Astronomy, The Hicks Building, University of Sheffield, Sheffield S3 7RH, UK \\ 7 Instituto de Astrofisica de Andalucia (CSIC), PO Box 3004, 18080 Granada, Spain \\ ${ }^{8}$ NASA Marshall Space Flight Center, NSSTC, XD-12, 320 Sparkman Drive, Huntsville, AL 35805, USA
}

Received 11 August 2005 / Accepted 3 September 2005

\section{ABSTRACT}

We present early WHT ISIS optical spectroscopy of the afterglow of gamma-ray burst GRB 050730. The spectrum shows a DLA system with the highest measured hydrogen column to date: $N(\mathrm{H} \mathrm{I})=22.1 \pm 0.1$ at the third-highest GRB redshift $z=3.968$. Our analysis of the Swift XRT $\mathrm{X}$-ray observations of the early afterglow show X-ray flares accompanied by decreasing X-ray absorption. From both the optical and the X-ray spectra we constrain the dust and gas properties of the host galaxy. We find the host to be a low metallicity galaxy, with low dust content. Much of the X-ray absorbing gas is situated close to the GRB, whilst the $\mathrm{H} \mathrm{I}$ absorption causing the DLA is most likely located further out.

Key words. gamma rays: bursts - galaxies: distances and redshifts - cosmology: observations

\section{Introduction}

Gamma-ray bursts (GRBs) have proven to be excellent probes of the distant Universe. High luminosity GRB afterglows allow absorption line studies of the ISM at high redshift to at least $z=4.5$ (see Andersen et al. 2000). The launch and successful operation of the Swift satellite means more GRBs are being localised and afterglows studied. Subsequently, the number of high redshift bursts suitable for host galaxy spectral studies has dramatically increased. Deep observations of afterglow positions have detected host galaxies in almost all cases (e.g. Conselice et al. 2005). Most hosts are compact, actively star-forming galaxies and, where the relevant data are available, are found to have low metallicity and low intrinsic extinction (e.g. Berger et al. 2003; Tanvir et al. 2004; Christensen et al. 2004). However, in a few cases, radio/submm observations of hosts give a star-formation rate (SFR) which is of order a few to $\sim 100$ times larger than rates derived from optical estimators such as the line luminosities of $\mathrm{H} \alpha$ and [O II] or the $2800 \AA$ restframe UV continuum flux (e.g. Berger et al. 2003). This may be caused by strong dust obscuration, but neither spectra nor colours of hosts show strong internal extinction. Afterglow spectroscopy provides a unique window on the near

* Table 1 is only available in electronic form at http://www. edpsciences.org environment of GRBs (e.g. GRB 021004, Schaefer et al. 2003; Fiore et al. 2005; Starling et al. 2005), allowing us to probe the absorbing dust and gas properties in more detail. In this Letter we present optical and X-ray spectra of GRB 050730, discovered by Swift on July 30th 2005, 19:58:23 UT (Holland et al. 2005) and lying at a redshift of $z=3.97$ (Chen et al. 2005a; Rol et al. 2005), in which we study the circumburst gas and dust properties.

\section{The optical afterglow spectra}

\subsection{Observations}

During the afterglow phase of GRB 050730, we acquired spectra using the Intermediate-dispersion Spectroscopic and Imaging System (ISIS) on the William Herschel Telescope. The R316R and R300B grisms were used on the red and blue arms respectively. Two observations were done sequentially, at the parallactic angle, with exposure times of 1260 and $1800 \mathrm{~s}$. The first observation started at 22:57 UT at airmass $\sim 2.73$ (midpoint 0.132 days after burst), the second at 23:19 UT (midpoint $0.145 \mathrm{~d}$ ) and airmass $\sim 3.4$. The seeing quality at the high airmasses required the slit width to be widened to 2.5 arcsec. Conditions during the observations were not photometric. These factors mean that our absolute flux calibration is not reliable, but the relative calibration should not be affected. 


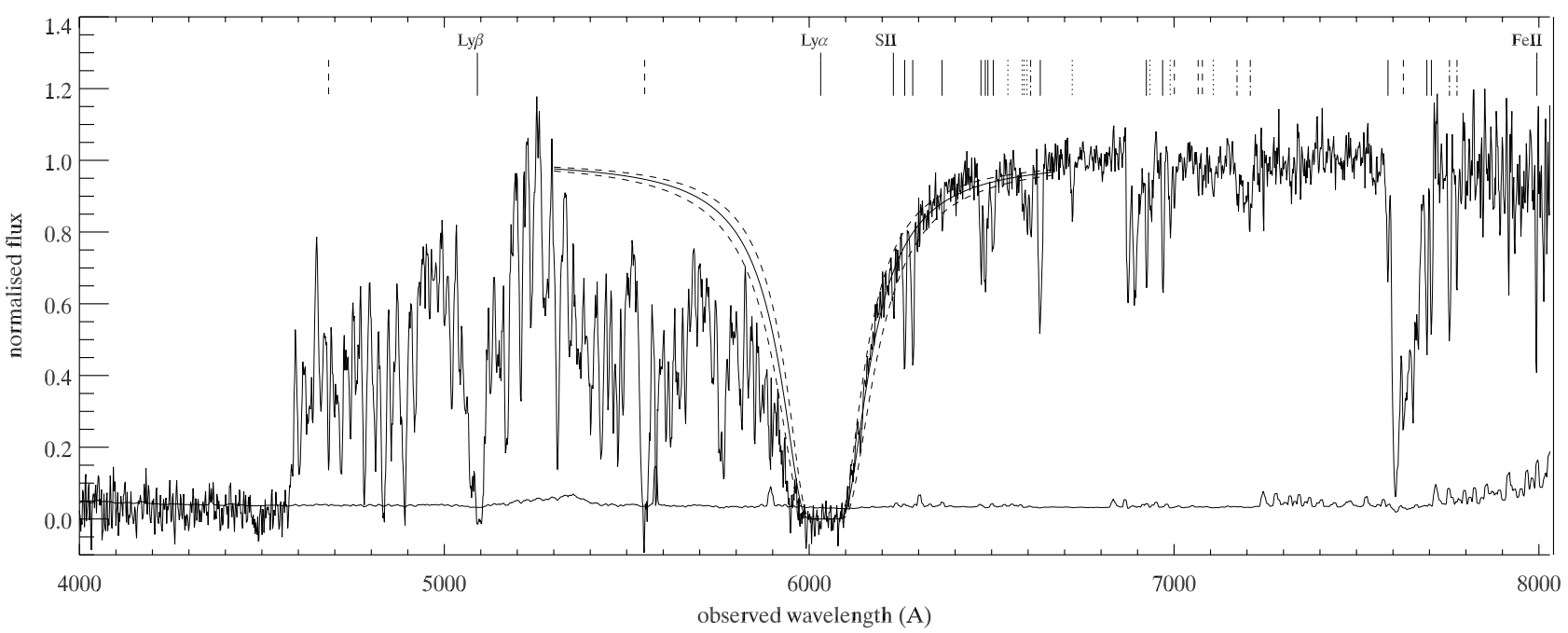

Fig. 1. The WHT ISIS combined, normalised spectrum of the afterglow (midpt 0.14 days), and $1 \sigma$ error spectrum (lower curve). Overlaid is the best-fitting DLA profile (solid line) and its errors (dashed lines). All significant lines ( $3 \sigma)$ are indicated above for $z=3.969$ (solid), $z=3.565$ (dashed), $z=1.773$ (dot-dashed) and unidentified (dotted) systems; see on-line table (Table 1) for details. Lines used in further analysis are labelled.

Both spectra have been reduced using the data reduction package IRAF following standard procedures. A Galactic extinction correction of $E(B-V)=0.049$ (Schlegel et al. 1998) was applied. The wavelength resolutions of blue- and red-arm spectra respectively are 8.7 and $8.1 \AA$. The signal to noise per pixel, measured at $6800 \AA$, is 27 in the first and 17 in the second spectrum.

\subsection{Results}

The spectrum, shown in Fig. 1, is rich in line features at $z=3.97,3.56$ and 1.77. A strong Damped Lyman-Alpha absorption system (DLA) is present; here we focus on this and a selection of metal lines presumed to originate in the GRB host galaxy. We fitted a power law continuum corrected for Galactic extinction to the $\sim 6500-7500 \AA$ region of each spectrum, excluding the absorption lines, and find an epoch averaged slope of $\beta=-1.34 \pm 0.21$ ( $2 \sigma$ formal fit error). We tested for any departure from a pure power law due to host-galaxy extinction: fitting MW, LMC and SMC extinction curves (Pei 1992) all result in epoch averaged $A_{\mathrm{V}}=0.01$. The optical/IR spectral slope from published $B V R I J$ photometry extrapolated to a common epoch using a temporal decay slope of 0.89 (Haislip et al. 2005; Holman et al. 2005; Cobb et al. 2005; Blustin et al. 2005) gives $\beta \sim-1$, consistent with the spectral analysis but not very constraining.

Despite the moderate dispersion of the ISIS 300 grisms, the damping wings of the host galaxy DLA are clearly visible. In fact, the determination of $N(\mathrm{H} \mathrm{I})$ in DLAs based on long slit spectra is considerably simpler than for echelles. Since the damped profile may extend over many spectral orders in a typical echelle, accurate combination and flux calibration can be troublesome. Using the Starlink software DIPSO, we determine $\log N(\mathrm{H} \mathrm{I})=22.1 \pm 0.1$ (see Fig. 1). Taking Ly $\beta$ into account did not lead to a more accurate determination of $N(\mathrm{H} \mathrm{I})$, and the error on our fit is dominated by uncertainties in the determination of the power law continuum. The $N(\mathrm{H} \mathrm{I})$ value is consistent with, although slightly lower than, that reported by Chen et al. (2005b). This high value (the first DLA to break the $10^{22}$ atoms $\mathrm{cm}^{-2}$ barrier) continues the trend amongst GRB DLAs towards very high neutral hydrogen columns (e.g. Jensen et al. 2001; Hjorth et al. 2003; Vreeswijk et al. 2004).

Although our spectra do not enable as detailed a study of the metal lines as is possible via echelle observations (e.g. Chen et al. 2005b), we briefly comment on a selection of these. Detection limits are quoted at the $3 \sigma$ level. Although we detect both S II $\lambda, \lambda 1253,1259$, both lines are likely to be at least partially saturated. In addition, the weaker S II $\lambda 1253$ line which potentially offers a better limit on $N(\mathrm{~S}$ II) is blended with another (unidentified) feature (Chen 2005, private communication). We determine an upper limit of $[\mathrm{S} / \mathrm{H}]<-2.0$ based on the absence of the weaker S II $\lambda 1250 \AA$ line $^{1}$, in good agreement with Chen et al. (2005b). Similarly, from the Fe II $\lambda 1608$ line which is partially saturated and the undetected Fe II $\lambda 1611$, we determine $-2.9<[\mathrm{Fe} / \mathrm{H}]<-1.9$.

A search for variability in line features between our two spectra, separated by $\sim 25$ min, revealed no significant changes (see on-line table for details), neither did the $N(\mathrm{H} \mathrm{I})$ column vary.

\section{The absorbed X-ray afterglow}

\subsection{Observations}

We have analysed the early Swift XRT data, to look for evidence of intrinsic absorption in the X-ray spectrum. The XRT data consist of Windowed Timing (WT) mode data for the first orbit (133 to $793 \mathrm{~s}$ after the trigger) and start of the second orbit, and Photon Counting (PC) mode data for later orbits. The data were reduced using the standard pipeline for

\footnotetext{
${ }^{1} \mathrm{~S}$ abundance of $\log (\mathrm{S} / \mathrm{H})+12=7.20($ Grevesse $\&$ Sauval 1998)
} 


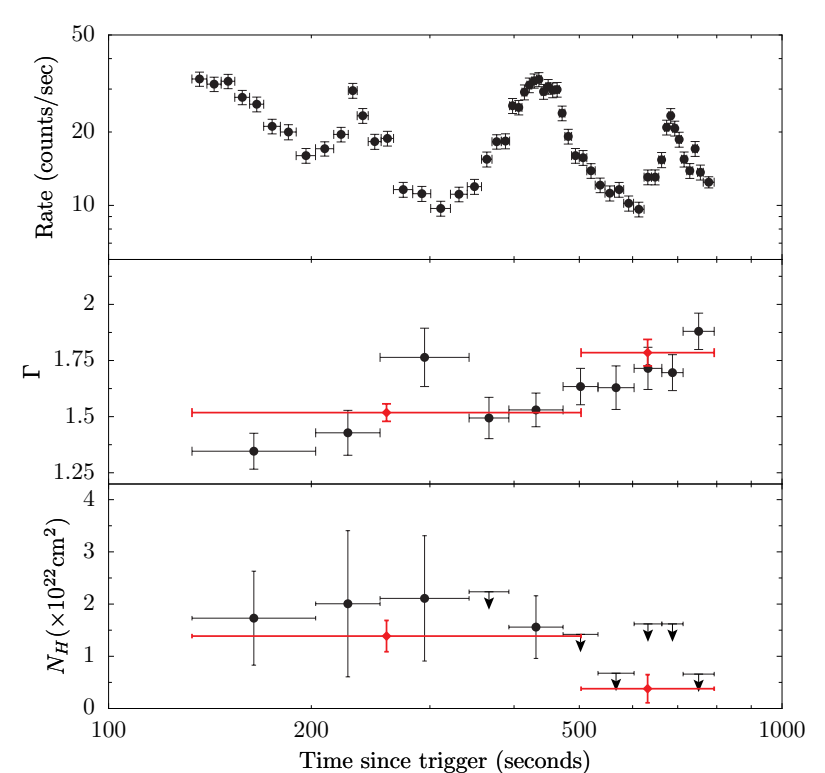

Fig. 2. Evolution of the $0.3-10 \mathrm{keV}$ count rate (top), $0.2-10 \mathrm{keV}$ power law slope, $\Gamma$, (middle, note that $\Gamma=1-\beta$ ) and additional equivalent hydrogen column, $N_{\mathrm{H}, \mathrm{int}}$, at the redshift of the host galaxy (bottom) during the first $\sim 800 \mathrm{~s}$ of the Swift XRT observations (90\% errors). The individual spectra are shown with filled circles; the two combined spectra pre- and post-500 s, for which better constraints are obtained, are shown with diamonds.

XRT data within the HEADAS 6.0 package (Swift software version 2.0). WT mode data was extracted using a rectangular region centred on the source, and a similar area in a source-free region of the same image to determine the background level. PC mode data was extracted using a circular aperture, except for orbits 2 to 4 which show evidence of pile-up (count rates $\gtrsim 0.8$ counts $\mathrm{s}^{-1}$ ) and were extracted using an annular region centred on the source and filtered on grade 0 only. The light curve was obtained between channels 30 and 1000 (spanning $\sim 0.3-10 \mathrm{keV}$ ). Spectral analysis was done using XSpec 11.3, with the standard Ancillary Response Function (ARF) files, which estimate the effective telescope area, for PC mode data, and with ARFs based on ray-tracing ("physical" ARFs) for WT mode data which should provide a better calibration at low energies.

\subsection{Results}

The first orbit shows several flares in the light curve, first reported for this afterglow by Grupe et al. (2005). We have performed a detailed analysis of the spectral evolution of the early-time data. The fitted model consists of a power law plus Galactic absorption (fixed at $3.05 \times 10^{20} \mathrm{~cm}^{-2}$, Dickey \& Lockman 1990) and a variable Galactic-like absorption component with Solar metallicity and $z=3.97$. Errors are quoted at the $90 \%$ confidence level for 1 interesting parameter. We find evidence for a change in power law photon index, from $\Gamma=1.52 \pm 0.04$ at the start of the first orbit to $1.79 \pm 0.06$ at the end of the orbit (note that $\Gamma=1-\beta$ ). We also find evidence for an excess absorption column, which at the redshift of the burst amounts to an intrinsic column of $N_{\mathrm{H} \text {,int }}=(1.4 \pm 0.3) \times$ $10^{22} \mathrm{~cm}^{-2}$. However, around $500 \mathrm{~s}$ post trigger, the absorption column abruptly changes, becoming lower by about a factor of 4: $N_{\mathrm{H} \text {,int }}=(3.4 \pm 2.7) \times 10^{21} \mathrm{~cm}^{-2}$. In the late-time PC mode spectrum the intrinsic column cannot be constrained, setting an upper limit of only $N_{\mathrm{H} \text {,int }} \leq 1.0 \times 10^{22} \mathrm{~cm}^{-2}$, and the power law photon index remains stable at $\Gamma \sim 1.77$. We have checked for a possible correlation between the intrinsic $N_{\mathrm{H} \text {,int }}$ and $\Gamma$ in the fit. Contour plots for the intervals 133-503 s and 503-793 s post trigger show no evidence for any correlation, confirming the reality of both the drop in $N_{\mathrm{H} \text {,int }}$ and increase in $\Gamma$ (Fig. 2). Interestingly, this happens directly after the peak of the second visible flare, where the light curve intensity has increased by a factor of 3 . Given the host galaxy metallicity we measure in the optical spectrum, we adjust the X-ray absorption model accordingly. Using $Z=Z_{\odot} / 100$ for all the elements heavier than He included in the zvphabs X-ray absorption model, the required intrinsic equivalent hydrogen column increases by a factor of $\sim 10$ in both cases to $N_{\mathrm{H} \text {,int }}=9.5_{-2.1}^{+2.3} \times 10^{22} \mathrm{~cm}^{-2}$ (first $400 \mathrm{~s})$ and $N_{\mathrm{H}, \text { int }}=2.6_{-1.6}^{+1.9} \times 10^{22} \mathrm{~cm}^{-2}(\geq 500 \mathrm{~s}$ post trigger $)$ with approximately the same goodness of fit.

A preliminary analysis of published optical photometry together with the PC mode XRT spectrum has shown the X-ray and optical slopes at 0.19 days $\left(\beta_{\mathrm{X}} \sim-0.7\right.$ to $-0.8, \beta_{\text {opt }} \sim-1.0$ to -1.5$)$ to likely be incompatible with a position of the cooling break between the optical and xrays, and might suggest the presence of an inverse Compton component; we await the availability of further optical/IR photometry for a full analysis.

\section{Discussion and conclusions}

\subsection{Host galaxy properties}

There is a well known relationship between galaxy luminosity and metallicity (e.g. Garnett 2002; Lamareille et al. 2004) which spans 6 orders or more of magnitude in $M_{B}$. Tremonti et al. (2004) have recently demonstrated that this relation is driven by an underlying relation between mass and metallicity. The cause of the relationship, they argue, is due to the increased gravitational potential of massive galaxies which enhances metal retention. In the absence of a detected host for GRB 050730 at the time of writing, it is in principle possible to use the luminosity-metallicity (LZ) and mass-metallicity (MZ) relations to predict the $M_{B}$ and stellar mass of the host. Both of these relations are best determined locally (e.g. Lamareille et al. 2004), although sizeable datasets have now investigated the LZ relation up to $z \sim 1$ (e.g. Kobulnicky et al. 2003; Kobulnicky \& Kewley 2004). There is clear evidence for evolution in the LZ relation, in the sense that galaxies are more metal-poor for their luminosity at higher $z$ (although see caveats in Kewley \& Ellison in prep.). This trend appears to continue both for the $\mathrm{LZ}$ and MZ relations up to $z \sim 3$ (e.g. Shapley et al. 2004; Møller et al. 2004; Erb et al. in prep.), although only the highest mass/luminosity galaxies are bright enough to be included in spectroscopic samples. The lowest metallicity bin in the fitted $\mathrm{MZ}$ relation of Erb et al. (in prep.) is $Z \sim Z_{\odot} / 3$ corresponding to a stellar mass $\log \left(M_{\star} / M_{\odot}\right) \sim 9.5$. The metallicity measured from absorption lines in the optical afterglow considered here is $Z \sim Z_{\odot} / 100$, which indicates that the host is not a massive, luminous Lyman break galaxy (LBG), although Jakobsson et al. (2005) argue that GRB hosts follow 
the same UV luminosity function as the faint LBGs. We do note that the MZ relation is based on emission lines. However, HST imaging has shown that GRBs occur in regions of strongest star formation (e.g. Fruchter et al. 2005), justifying our assumption that the absorption lines are formed in the same regions as the higher wavelength emission lines. Combining the measured $N(\mathrm{H} \mathrm{I})$ with the metallicity and assuming an SMC gas-to-reddening ratio (Bouchet et al. 1985), we can estimate the extinction associated with the GRB host galaxy and compare this to the values obtained from the optical continuum fits. Negligible $E(B-V)$ is determined by both methods, consistent with the similarly small amounts of dust seen towards intervening DLAs (e.g. Murphy \& Liske 2004; Ellison et al. 2005).

\subsection{The neutral hydrogen column}

GRB 050730 has the strongest DLA seen in a GRB afterglow spectrum, with a hydrogen column density of $\log N(\mathrm{H} \mathrm{I})=$ $22.1 \pm 0.1$. The X-ray absorption at late times scaled to $Z_{\odot} / 100$ yields a comparable $\log N_{\mathrm{H} \text {,int }}=22.4_{-0.4}^{+0.2}$ (assuming the $N_{\mathrm{H} \text {,int }}$ measured at $\sim 500-800$ s post burst in the WT mode XRT spectrum can be extrapolated to a few hours post burst). The $N_{\mathrm{H} \text {,int }}$ we measure in the early-time X-ray spectra covering $\sim 133-500$ s post trigger is about ten times higher than that measured at $t>500 \mathrm{~s}$. The change in X-ray absorbing column could be caused by ionisation by the gamma-ray jet, or by the $\mathrm{X}$-ray flares which are suggested to be caused by prolonged central engine activity (Burrows et al. 2005; King et al. 2005).

It should be noted that what is measured in the X-ray models is an equivalent hydrogen column, since primarily metal edges contribute to the X-ray absorption at the redshift of GRB 050730, and that this is highly dependent upon the metallicity assumed (see e.g. Wilms et al. 2000). There will be a contribution to the X-ray absorption from intervening systems, which cannot be disentangled from absorption in the host, particularly given that we do not know the metallicity of the closest intervening system observed in this spectrum $(z=1.77)$. In principle, a lower column very close to the observer could have a similar effect on the spectrum as a large column at high redshift.

The observed X-ray column variability does, however, lead us to conclude that most of the X-ray absorbing gas in GRB 050730 is located close to the GRB. The optical H I column remained stable over the $\sim 25$ min between our ISIS spectra, taken at 0.132 days since burst, well after the observed X-ray flaring (although the occurrence of X-ray flares at later times cannot be ruled out owing to low count rates). The $\mathrm{H}$ I creating the DLA is likely to be located much further away from the GRB, unaffected by the GRB radiation. We would expect to observe destruction by the GRB of dust co-located with the X-ray absorbing gas. Our spectra imply a very low extinction in the host at $\sim 3 \mathrm{~h}$ post burst. Future prompt optical spectra, in conjunction with X-ray observations, are required to investigate this further.

Acknowledgements. We thank J. P. U. Fynbo for comments on and improvements to the manuscript and P. A. Curran, A. J. van der Horst, K. L. Page and S. Vaughan for useful discussions. This work is based on observations made with the WHT operated on the island of La Palma by the Isaac Newton Group in the Spanish Observatorio del Roque de los Muchachos at the Instituto de Astrofisica de Canarias - we thank N. O'Mahony for excellent support. The authors acknowledge support from and collaboration within the EU-funded Research Training Network "Gamma-Ray Bursts: an enigma and a tool” (HPRN-CT-2002-00294).

\section{References}

Andersen, M. I., Hjorth, J., Pedersen, H., et al. 2000, A\&A, 364, L54 Berger, E., Cowie, L. L., Kulkarni, S. R., et al. 2003, ApJ, 588, 99 Blustin, A., Holland, S. T., Cucchiara, A., et al. 2005, GCN Circ. 3717 Bouchet, P., Lequeux, J., Maurice, E., et al. 1985, A\&A, 149, 330

Burrows, D. N., Romano, P., Falcone, A., et al. 2005, Science, accepted [arXiv:astro-ph/0506130]

Chen, H.-W., Thompson, I., Prochaska, J. X., et al. 2005a, GCN Circ., 3709

Chen, H.-W., Prochaska, J. X., Bloom, J. S., \& Thompson, I. B. 2005b, ApJL submitted [arXiv:astro-ph/0508270]

Christensen, L., Hjorth, J., \& Gorosabel, J. 2004, A\&A, 425, 913

Cobb, B. E., \& Bailyn, C. D., et al. 2005, GCN Circ., 3708

Conselice, C. J., Vreeswijk, P. M., Fruchter, A. S., et al. 2005, ApJ, preprint doi:10.1086/'432829'

Dickey, J. M., \& Lockman, F. J. 1990, ARA\&A, 28, 215

Ellison, S. L., Hall, P. B., \& Lira, P. 2005, AJ, in press [arXiv:astro-ph/0507418]

Fiore, F., D'Elia, V., Lazzati, D., et al. 2005, ApJ, 624, 853

Fruchter, A., et al. 2005, ApJ, submitted

Garnett, D. R. 2002, ApJ, 581, 1019

Grevesse, N., \& Sauval, A. J. 1998, Space Sci. Rev., 85, 161

Grupe, D., Kennea, J. A., \& Burrows, D. N. 2005, GCN Circ., 3714

Haislip, J., Kirschbrown, J., Reichart, D., et al. 2005, GCN Circ., 3712

Hjorth, J., Møller, P., Gorosabel, J., et al. 2003, ApJ, 597, 699

Holland, S. T., Barthelmy, S., Burrows, D. N., et al. 2005, GCN Circ., 3704

Holman, M., Garnavich, P., \& Stanek, K. Z. 2005, GCN Circ., 3727

Jensen, B. L., Fynbo, J. U., Gorosabel, J., et al. 2001, A\&A, 370, 909

Jakobsson, P., Bjornsson, G., Fynbo, J. P. U., et al. 2005, MNRAS, in press [arXiv:astro-ph/0505542]

King, A., O'Brien, P. T., Goad, M. R., et al. 2005, ApJL, accepted [arXiv:astro-ph/0508126]

Kobulnicky, H. A., \& Kewley, L. J. 2004, ApJ, 617, 240

Kobulnicky, H. A., Willmer, C. N. A., Weiner, B. J., et al. 2003, ApJ, 599, 1006

Lamareille, F., Mouhcine, M., Lewis, I., et al. 2004, MNRAS, 350, 396

Møller, P., Fynbo, J. P. U., \& Fall, S. M. 2004, A\&A, 422, L33

Murphy, M. T., \& Liske, J. 2004, MNRAS, 345, L31

Pei, Y. 1992, ApJ, 395, 130

Rol, E., Starling, R. L. C., Wiersema, K., et al. 2005, GCN Circ., 3710

Schaefer, B. E., Gerardy, C. L., Höflich, P., et al. 2003, ApJ, 588, 387

Schlegel, D. J., Finkbeiner, D. P., \& Davis, M. 2004, ApJ, 500, 525

Shapley, A. E., Erb, D. K., Pettini, M., et al. 2004, ApJ, 612, 108

Sota, A., Castro-Tirado, A. J., Guziy, S., et al. 2005, GCN Circ., 3705

Starling, R. L. C., Wijers, R. A. M. J., Hughes, M. A., et al. 2005, MNRAS, 360, 305

Tanvir, N. R., Barnard, V. E., Blain, A. W., et al. 2004, MNRAS, 352, 1073

Tremonti, C., Heckman, T. M., Kauffmann, G., et al. 2004, ApJ, 613, 898

Vreeswijk, P. M., Ellison, S. L., Ledoux, C., et al. 2004, A\&A, 419, 927

Wilms, J., Allen, A., \& McCray, R. 2000, ApJ, 542, 914 
R. L. C. Starling et al.: Gas and dust around GRB 050730, Online Material p 1

\section{Online Material}


R. L. C. Starling et al.: Gas and dust around GRB 050730, Online Material p 2

Table 1. Lines detected above $3 \sigma$ in the first and second epoch WHT spectra ${ }^{a}$.

\begin{tabular}{|c|c|c|c|c|}
\hline$\lambda$ & $W_{\text {obs }}(\mathrm{I})$ & $W_{\text {obs }}(\mathrm{II})$ & ID & $z$ \\
\hline $4683.0^{b}$ & & & $\operatorname{Ly} \beta \lambda 1025.722$ & 3.5656 \\
\hline $5090.6^{b}$ & & & $\operatorname{Ly} \beta \lambda 1025.722$ & 3.9629 \\
\hline $5549.2^{b}$ & & & $\operatorname{Ly} \alpha \lambda 1215.668$ & 3.5647 \\
\hline $6031.5^{b}$ & & & $\operatorname{Ly} \alpha \lambda 1215.668$ & 3.9615 \\
\hline 6230.8 & $1.42 \pm 0.24$ & $1.19 \pm 0.38$ & S II $\lambda 1253.805$ & 3.9695 \\
\hline \multirow[t]{2}{*}{6261.9} & $3.64 \pm 0.25$ & $3.50 \pm 0.41$ & Si II $\lambda 1260.422$ & 3.9681 \\
\hline & & blended with & S II $\lambda 1259.518$ & 3.9717 \\
\hline 6283.9 & $3.95 \pm 0.23$ & $3.45 \pm 0.33$ & Si II* $\lambda 1264.738$ & 3.9685 \\
\hline 6364.2 & $0.51 \pm 0.17$ & $0.78 \pm 0.35$ & C I? $\lambda 1280.135$ & 3.9715 \\
\hline 6470.8 & $1.95 \pm 0.17$ & $2.43 \pm 0.39$ & O I $\lambda 1302.169$ & 3.9692 \\
\hline \multirow[t]{2}{*}{6482.8} & $2.57 \pm 0.14$ & $2.87 \pm 0.28$ & $\mathrm{O} \mathrm{I}^{*} \lambda 1304.858$ & 3.9682 \\
\hline & & blended with & Si II $\lambda 1304.370$ & 3.9700 \\
\hline 6489.8 & $0.50 \pm 0.11$ & $0.82 \pm 0.20$ & $\mathrm{O} \mathrm{I}^{* *} \lambda 1306.029$ & 3.9691 \\
\hline \multirow[t]{2}{*}{6504.6} & $1.86 \pm 0.16$ & $2.17 \pm 0.35$ & Si II* $\lambda 1309.276$ & 3.9681 \\
\hline & & blended with? & Fe II $\lambda 2344.214$ & 1.7747 \\
\hline 6544.6 & $0.44 \pm 0.13$ & $0.47 \pm 0.17$ & & \\
\hline 6583.9 & $0.42 \pm 0.10$ & $0.55 \pm 0.18$ & & \\
\hline 6588.9 & $0.55 \pm 0.11$ & $0.57 \pm 0.17$ & & \\
\hline 6597.1 & $0.82 \pm 0.12$ & $1.33 \pm 0.26$ & & \\
\hline 6607.0 & $1.24 \pm 0.14$ & $1.58 \pm 0.23$ & Fe II $\lambda 2382.765$ & 1.7728 \\
\hline \multirow[t]{2}{*}{6633.4} & $5.12 \pm 0.19$ & $5.07 \pm 0.31$ & C II $\lambda 1334.532$ & 3.9706 \\
\hline & & blended with & $\mathrm{C} \mathrm{II}^{*} \lambda 1335.663$ & 3.9664 \\
\hline 6721.0 & $1.05 \pm 0.17$ & $0.73 \pm 0.20$ & & \\
\hline 6924.1 & $2.43 \pm 0.15$ & $2.42 \pm 0.32$ & Si IV $\lambda 1393.760$ & 3.9679 \\
\hline 6934.0 & $0.88 \pm 0.09$ & $1.18 \pm 0.24$ & & \\
\hline \multirow[t]{2}{*}{6968.7} & $2.67 \pm 0.15$ & $2.32 \pm 0.28$ & Si IV $\lambda 1402.773$ & 3.9678 \\
\hline & & blended with & Si II $\lambda 1526.707$ & 3.5645 \\
\hline 6989.4 & $1.44 \pm 0.17$ & $1.28 \pm 0.22$ & & \\
\hline 7000.9 & $0.52 \pm 0.09$ & $0.86 \pm 0.24$ & $\mathrm{Si} \mathrm{II}^{*} \lambda 1533.432$ & 3.5655 \\
\hline 7066.1 & $0.42 \pm 0.12$ & $0.20 \pm 0.19$ & $\operatorname{CIV} \lambda 1548.204$ & 3.5641 \\
\hline 7077.7 & $0.32 \pm 0.12$ & $0.48 \pm 0.18$ & $\operatorname{CIV} \lambda 1550.781$ & 3.5640 \\
\hline 7107.4 & $0.62 \pm 0.14$ & $0.61 \pm 0.22$ & & \\
\hline 7172.6 & $0.71 \pm 0.14$ & $0.92 \pm 0.25$ & Fe II $\lambda 2586.650$ & 1.7729 \\
\hline 7209.0 & $1.46 \pm 0.17$ & $0.86 \pm 0.20$ & Fe II $\lambda 2600.173$ & 1.7725 \\
\hline $7586.4^{c}$ & $2.53 \pm 0.18$ & $2.48 \pm 0.26$ & Si II $\lambda 1526.707$ & 3.9691 \\
\hline $7628.5^{c}$ & & & Al II $\lambda 1670.789$ & 3.5658 \\
\hline 7692.9 & $3.79 \pm 0.14$ & $3.86 \pm 0.29$ & C IV $\lambda 1548.204$ & 3.9689 \\
\hline 7705.3 & $3.66 \pm 0.20$ & $4.22 \pm 0.36$ & $\mathrm{C}$ IV $\lambda 1550.781$ & 3.9687 \\
\hline 7754.9 & $4.18 \pm 0.34$ & $5.15 \pm 0.63$ & $\operatorname{Mg}$ II $\lambda 2798.743$ & 1.7709 \\
\hline 7775.5 & $2.28 \pm 0.25$ & $2.67 \pm 0.36$ & $\operatorname{Mg}$ II $\lambda 2803.532$ & 1.7735 \\
\hline 7994.3 & $3.14 \pm 0.54$ & $3.27 \pm 0.74$ & Fe II $\lambda 1608.451$ & 3.9716 \\
\hline
\end{tabular}

a Blueward of $\operatorname{Ly} \alpha$ the low resolution and $\operatorname{Ly} \alpha$ forest hampers secure identification of metal lines, which we therefore do not list.

${ }^{b}$ Due to the uncertain continuum level, we do not attempt to measure the widths of the $\operatorname{Ly} \alpha$ and $\operatorname{Ly} \beta$ lines.

${ }^{c}$ This equivalent width measurement is seriously affected or made impossible by the atmospheric absorption band from 7584-7675 A. 